\title{
Quantum ergodicity for electrons in two dimensions
}

\author{
Dima L. Shepelyansky ${ }^{1}$, and Pil Hun Song ${ }^{2,3}$ \\ ${ }^{1}$ Laboratoire de Physique Quantique, UMR 5626 du CNRS, Université Paul Sabatier, \\ F-31062 Toulouse Cedex 4, France \\ ${ }^{2}$ Center for Theoretical Physics, Seoul National University, \\ Seoul 151-742, Korea \\ 3 Max-Planck-Institut für Kernphysik, Postfach 103980, \\ 69029 Heidelberg, Germany \\ dima@irsamc2.ups-tlse.fr
}

Received 29 July 1999, accepted xxxxxx by xxx

\begin{abstract}
We study the effect of electron-electron interaction on a two dimensional (2D) disordered lattice. For the case of two electrons the analytical estimates are presented showing a transition from localized to delocalized states in a way similar to the Anderson transition in $3 \mathrm{D}$. The localized phase corresponds to large values of the parameter $r_{s}$, which is determined by the ratio of the Coulomb and Fermi energies. The numerical investigations of the spectral statistics $P(s)$ in a system with up to $N_{p}=30$ spin polarized electrons show the transition from the Poisson to the Wigner-Dyson distribution at a total electron energy independent of $N_{p}$. The relation to experiments on the metal-insulator transition in $2 \mathrm{D}$ is also discussed.
\end{abstract}

Keywords: metal-insulator transition, interactions, quantum chaos

PACS: $71.30 .+\mathrm{h}$, 72.15.Rn, 05.45.Mt

\section{Introduction}

Since 1979 it became clear that non-interacting electrons are always localized in a 2D disordered potential [1]. At present this result is firmly confirmed by different analytical and numerical studies (see the reviews [2, 3]) according to which all one-particle eigenstates are exponentially localized in the case of orthogonal or unitary symmetry. Delocalization is possible only for the symplectic symmetry which is however not realized in the absence of spin-orbit interaction [2, 3]. Therefore, the discovery of metallic behavior in 2D high mobility samples by Kravchenko et. al. [4] attracted a great interest of the solid-state community and pushed forward the question about a role of electron-electron interactions in the localized phase. Indeed, in many experiments of different groups 4, 5, 6, 7, 8, 9, 10, 11, 12, the ratio of the Coulomb interaction energy $E_{e e}$ to the Fermi energy $\epsilon_{F}$ is characterized by a large dimensionless parameter $r_{s}=1 / \sqrt{\pi n_{s}} a_{B}^{*} \simeq E_{e e} / \epsilon_{F}$, where $n_{s}$ is the electron density in $2 \mathrm{D}$, and $a_{B}^{*}=\hbar^{2} \epsilon_{0} / m^{*} e^{2}, m^{*}, \epsilon_{0}$ are the effective Bohr radius, electron mass and dielectric constant, respectively. Typically in the above experiments the parameter $r_{s}$ is ranged in the interval 5 - 40 showing that these experiments are well outside of the perturbative regime corresponding to $r_{s} \ll 1$. Generally, in these experiments the insulating increase of resistivity at low temperature $T$ is observed at large $r_{s}$ values (low charge 
density $n_{s}$ ), while its metallic decrease at low $T$ is seen at lower $r_{s}$ (higher $n_{s}$ ). In addition, experimentally it was found that a sufficiently strong magnetic field parallel to the $2 \mathrm{D}$ plane destroys the metallic behavior 13, 14. These results show an important role of the spin degrees of freedom. However, we should note that the recent experiments [15] with n-SiGe samples demonstrate that the metallic behavior persists even in very strong in plane magnetic field when all spins are polarized. Another important experimental indication was found for a localized phase with a variable range hopping (VRH) 16, 17]. Indeed, the experiments show that a prefactor in the exponential VRH resistivity dependence on temperature is phonon independent [17]. For the theoretical explication of this result other physical mechanisms of VRH should be found [18] and it is possible that their origin is related to the electron-electron interaction as it had beed discussed long ago in 19 .

From the theoretical view point the problem of interaction in the localized phase is rather nontrivial. Indeed, due to localization of noninteracting states, the two interacting particles (TIP), with a short range repulsive/attractive interaction and located on a distance of one-particle localization length $l_{1}$ from each other, always return to their center of mass that enhances enormously their interaction. As a result the two particles can propagate coherently on a distance much larger $l_{1}$. This effect has been discussed recently by different groups for a short range interaction 20, 21, 22, 23. 24, 25] (see also the early paper by Dorokhov for a strongly attractive case [26]). However, in the experiments discussed above the charge density is very low and the distance between electrons (holes) is much larger than $a_{B}^{*}\left(r_{s} \gg 1\right)$. In this situation the localized electrons interact via the long range Coulomb interaction. Recently, it has been shown [27, 28] that in this situation two interacting electrons can be delocalized even when they are separated by a distance $R \gg l_{1}$. Moreover, this delocalization goes in a way very similar to the Anderson transition in $3 \mathrm{D}$. In this paper we discuss the physical origin of this delocalization (Section II). After that (Section III) we present the results of extensive numerical studies of up to $N_{p}=30$ electrons (with polarized spins) on 2D disordered lattice. In agreement with the first results presented in 29] we establish that the ground state remains localized (nonergodic) but at moderate values of parameter $r_{s}$ the electrons become ergodic at very low total energy where their spectral statistics demonstrates a transition from the Poisson to the Wigner-Dyson distribution. The conclusion is presented in Section IV.

\section{Two interacting electrons in the 2D Anderson model}

Let us consider a system of electrons with polarized spins on a 2D disordered lattice (Anderson model). Such polarization can be reached by a strong in plane magnetic field but also one can speak about spinless fermions. The system is described by the Hamiltonian

$$
H=V \sum_{<i j>} a_{i}^{\dagger} a_{j}+\sum_{i} w_{i} n_{i}+U \sum_{i>j} \frac{n_{i} n_{j}}{r_{i j}} .
$$

Here $a_{i}^{\dagger}\left(a_{i}\right)$ is the fermion creation (annihilation) operator at site $i$, the hopping between the nearest neighbors is $V$, the diagonal energies $w_{i}$ are randomly distributed 
within the interval $[-W / 2, W / 2]$ and $U$ is the strength of the Coulomb interaction with $r_{i j}$ being the distance between electrons at sites $i, j$. In this notation $n_{i}=a_{i}^{\dagger} a_{i}$ is the occupation number at site $i$. The electrons (particles) are moving in a 2D cell of size $L \times L$ with periodic boundary conditions. The Coulomb interaction is taken between electrons in one cell of size $L$ and with 8 charge images in nearby 8 cells as in 27. The number of particles $N_{p}$ and the cell size were varied within the intervals $2 \leq N_{p} \leq 30$ and $8 \leq L \leq 31$. With the notations of Eq. (1), the parameter $r_{s}$ is given by $r_{s}=U /(2 V \sqrt{\pi \nu})$, where $\nu=N_{p} / L^{2}$ is the filling factor and $\epsilon_{F}=4 \pi \nu V$. The majority of our data have been obtained for $U / V=2,0.0048<\nu<0.03$ that corresponds to $3.22<r_{s}<8.14$. When changing $N_{p}$ the filling factor was kept approximately constant (nearest rational value) by the appropriate choice of $L$.

For $U=0$ all states in the Hamiltonian (1) are localized and the one-particle localization length varies exponentially with $W$ in the limit of weak disorder: $\ln l_{1} \sim$ $(V / W)^{2}$. The numerical diagonalization [27, 28] allows to determine the inverse participation ratio (IPR) for one particle $\xi_{1}$, which approximately gives the number of lattice sites contributing in one eigenstate. The values found at $L=24$ for different disorder strength at the ground state and the middle of the band are correspondingly: $\xi_{1}=3.4$ and $4.2(W / V=15) ; 5.2$ and $11.2(W / V=10) ; 8.2$ and $36.7(W / V=7)$; 13.5 and $84.2(W / V=5)$. This shows that the one-particle states at low energy and even at the band center (except maybe $W / V=5)$ are well localized $\left(\xi_{1} \ll L^{2}\right)$.

To understand how two electrons interact in the $2 \mathrm{D}$ localized phase we should estimate the interaction induced transition rate $\Gamma_{2}$ between unperturbed $(U=0)$ localized eigenstates. Following [27, 28] let us assume that the distance between electrons $R$ is much larger than $l_{1}\left(R \gg l_{1}\right)$ and their energy is in the middle of the band. In this case the two-body interaction has a dipole-dipole form since the lower order terms only slightly modify the one-particle effective potential. The two-body matrix element between noninteracting eigenstates is then $U_{s} \sim U l_{1}^{2} / R^{3} \times \sum \psi^{4}$, where we used that the dipole is of the order of $l_{1}$ (electron cannot jump on a distance larger $l_{1}$ due to exponential localization). The sum $\sum$ of the product of four random one-particle wave functions $\psi \sim 1 / l_{1}$ runs over $l_{1}^{2}$ sites for each electron so that $\sum \sim 1 / l_{1}^{2}$ and $U_{s} \sim U / R^{3}$. The density of directly coupled two electron states is $\rho_{2} \sim l_{1}^{4} / V$ and according to the Fermi golden rule the hopping rate is $\Gamma_{2} \sim U_{s}^{2} \rho_{2} \sim U^{2} l_{1}^{4} /\left(V R^{6}\right)$. The mixing of two-electron levels takes place when $\kappa_{e}=\chi_{e}^{2} \sim \Gamma_{2} \rho_{2} \sim\left(r_{L}^{4 / 3} / r_{s}\right)^{2}>1$, where $r_{L}$ is the value of $r_{s}$ at the filling $\nu=1 / l_{1}^{2}$ (one electron in a box of size $l_{1}$ with $\left.r_{L}=l_{1} U /(2 \sqrt{\pi} V)\right)$. For $U \sim V$ this mixing takes place at $R \sim l_{1}^{4 / 3} \gg l_{1}$. In this sense the situation is qualitatively different from the case of short range interaction where the mixing is possible only for particles on a distance $R<l_{1}$. For $\kappa_{e}>1$ the pair of two electrons becomes delocalized in a way similar to the 3D Anderson transition. Indeed, the center of mass can move in the $2 \mathrm{D}$ plane and in addition electrons can rotate around a ring of radius $R$ and width $l_{1}$ keeping their total energy $E \sim U / R$ constant. Since $R>>l_{1}$ the dynamics is going in an effective 3D space where the Anderson transition to diffusion takes place at $\kappa_{e}>1$. Formally, the diffusion of two electrons will be finally localized since the finite length of the ring makes the situation analogous to quasi-2D Anderson model (finite number of planes). However, the corresponding TIP localization length $l_{c}$ jumps from $l_{c} \sim l_{1}$ at $\kappa_{e}<1$ to exponentially large 
$l_{c} \sim l_{1} \exp \left(\pi l_{1}^{1 / 3} \kappa_{e}\right) \gg l_{1}$ for $\kappa_{e}>1$. In a similar way for $M$ coupled 2D Anderson models the one-particle localization length $l_{1}$ makes a jump near the 3D transition point (e.g. $W / V=16.5)$ from $l_{1} \sim 1$ to $l_{1} \sim \exp (g)$ where the conductance $g \sim M$. In the delocalized phase the diffusion rate or the conductivity in units $e^{2} / h$ can be estimated as $D_{e} \sim l_{1}^{2} \Gamma_{e} \sim V \kappa_{e} / l_{1}^{2}$. The above estimates show that the border between the insulating (localized at $r_{s}>r_{L}^{4 / 3}$ ) and metallic (delocalized at $r_{s}<r_{L}^{4 / 3}$ ) phases is:

$$
r_{s} \sim r_{L}^{4 / 3}
$$

The physical meaning of this result is very natural: larger $r_{s}$ corresponds to a lower density $n_{s}$ with larger distance between electrons and smaller two-body interaction between them.

The first numerical studies of two polarized electrons in 2D Anderson model showed a transition in the level spacing statistics $P(s)$ from the Poisson distribution $P_{P}(s)$ near the ground state to the Wigner-Dyson statistics $P_{W}(s)$ at higher energies 27] in a way similar to the results of Shklovskii et al. [30] for the 3D Anderson model. In both cases the intermediate statistics $P(s)$ are very close at the critical transition point (Fig. 2 in [27]). The numerical studies were done in the range of parameters $5 \leq W / V \leq 15,0.1 \leq U / V \leq 2,6 \leq L \leq 24, r_{s}<10$; more details can be found in [27, 28. Independent recent studies [31] of a similar model with Coulomb interaction at the center of the energy band also show a transition to $P_{W}(s)$ as a function of $W$. Since even analytical formulas for the Coulomb matrix element between localized states are absent further investigations of this problem are still needed.

\section{Numerical studies of multi-electron problem in 2D}

For numerical studies of the Hamiltonian (1) we follow the approach developed in [27, 29] and rewrite the hamiltonian matrix in the basis of one particle eigenstates (orbitals) at $U=0$ using the computed two-body matrix elements between the orbitals. Only a finite number $M$ of low energy orbitals has been considered and the final manybody hamiltonian matrix was constructed on the basis of a pyramid rule for the oneparticle orbital index $m_{i}: \sum_{i=1}^{N_{p}} m_{i} \leq \sum_{i=1}^{N_{p}-1} i+M$. This procedure allows to make an efficient reduction of the resulting matrix size $N_{m}$ comparing to ${ }_{M} C_{N_{p}}$ without any serious modification of the properties of low energy states. To the maximum we used $N_{m} \approx 5000$ corresponding to $N_{p}=20, M=42$ (for $N_{p}=30$ we used up to $M=49$ ). We checked that our results at low energy are not sensitive to the variation of $M$ and $N_{m}$ (see the illustrations below). One of the main characteristics we extracted from the numerical studies is the level spacing statistics $P(s)$ at a given total excitation energy $E$ measured from the ground state. The disorder average has been performed over $N_{D}=5000$ realization (for low $E$ ) and $N_{D}=1000$ (for higher $E$ ). In this way, the total statistics used for $P(s)$ varied from $N_{s}=10^{4}$ at low $E$ to $N_{S}=3 \times 10^{5}$ at high $E$. To study the variation of $P(s)$ with the interaction or disorder strength it is convenient to use the parameter $\eta$ which is defined as $\eta=\int_{0}^{s_{0}}\left(P(s)-P_{W}(s)\right) d s / \int_{0}^{s_{0}}\left(P_{P}(s)-P_{W}(s)\right) d s$, where $s_{0}=0.4729 \ldots$ is the smaller intersection point of $P_{P}(s)$ and $P_{W}(s)$. In this way $\eta=1$ corresponds to $P_{P}(s)$ and 


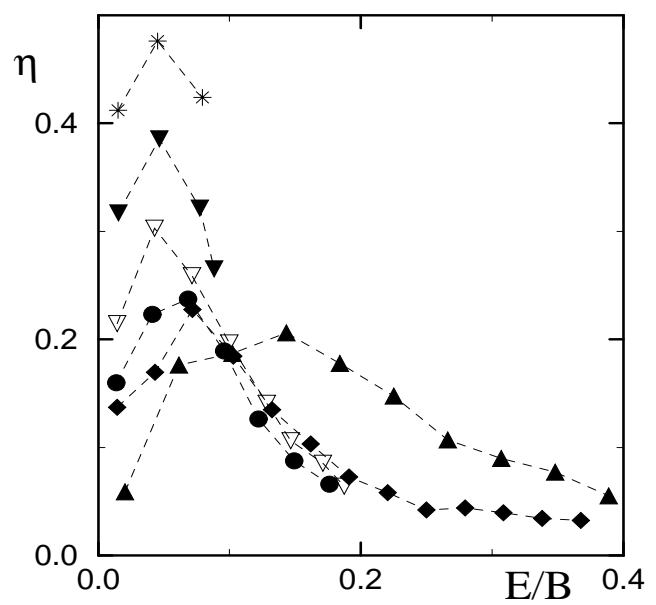

Fig. 1 Dependence of $\eta$ on the rescaled total energy $E / B$ for various numbers of particles $N_{p}=3$ (full triangle up), 6 (full diamond), $10(\bullet), 12$ (triangle down), 20 (full triangle down) and $30\left(^{*}\right) ; W / V=5$, filling factor $\nu \approx 1 / 32$ and $r_{s}=3.22,10 \leq L \leq 31 ; B=4 V$.

$\eta=0$ to $P_{W}(s)$. We remind that for the one-particle 3D Anderson model the localized phase is characterized by $\eta=1$, since localized (nonergodic) eigenstates do not feel each other, while in the metallic phase the eigenstates are extended and $\eta=0$ [30].

The results presented in 29] show that at $r_{s}=3.22$ the parameter $\eta$ evolves to 1 for the total energy $E<E_{c}$ and 0 for $E>E_{c}$ with the growth of the system size $L$ at fixed filling $\nu \approx 1 / 32$. The critical energy $E_{c}$ and $\eta_{c}$ at which the transition takes place are $E_{c} \approx 0.25 B, \eta_{c} \approx 0.56(W / V=10)$ and $E_{c} \approx 0.15 B, \eta_{c} \approx 0.33(W / V=7)$ with $B=4 V$. For $W / V=15$ no transition is found for $E / B \leq 1$ where $\eta \approx 0.8-1.0$. The new data for $W / V=5$ are presented in Fig. 1 where the transition is seen at $E_{c} \approx 0.1 B, \eta_{c} \approx 0.19$. At the critical point $\left(E_{c}, \eta_{c}\right)$ the spectral statistics $P(s)$ is independent of the number of particles and the system size reached in our simulations (see Fig. 3 in 291). In Fig.2 we show an illustration that at low energy the parameter $\eta$ is not sensitive to the variation of the total matrix size $N_{m}$ by more than 3 times that confirms the validity of our numerical approach. We note that not only the values of $\eta$ are close for different $N_{m}$ but also the whole distributions $P(s)$, as it is illustrated in Fig. 3 for $r_{s}=5.76$.

The results of Fig. 1 are obtained at fixed parameter $r_{s}$. The variation of the spectral statistics $P(s)$ at low energy for the change of $r_{s}$ from 3.2 to 8.14 is shown in Fig. 3. These data demonstrate a clear approach to the Poisson distribution with growing $r_{s}$. The same tendency is seen in the $\eta$ dependence on energy shown at different $r_{s}$ in Fig. 4. These results are in agreement with the relation (2) and the argument given in the previous section according to which at large $r_{s}$ the distance between electrons grows and the two-body interaction between them drops. Since 


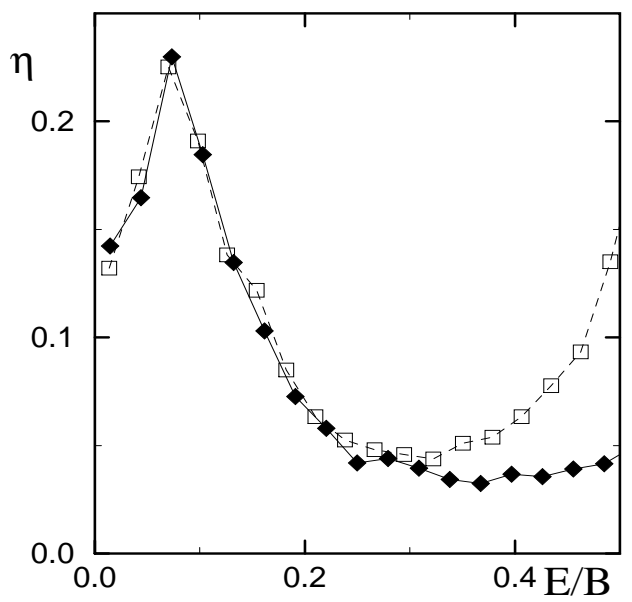

Fig. 2 Dependence of $\eta$ on $E / B$ for the case $N_{p}=6$ of Fig.1 for different matrix sizes: $N_{m}=1513, M=26$ (full diamond); $N_{m}=498, M=21$ (square); here $N_{D}=5000$.

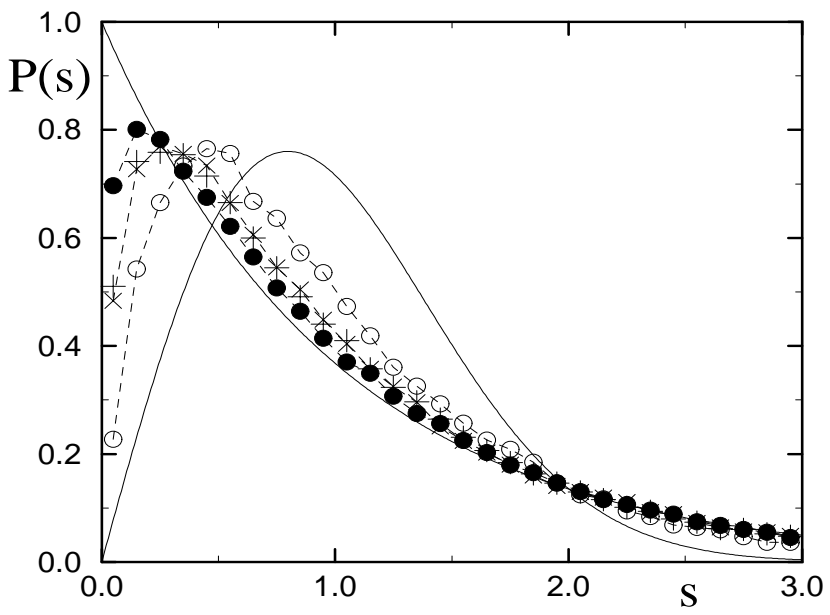

Fig. 3 Level statistics $P(s)$ at $W / V=7, L=25$ in the energy interval $0.1<E / B<0.14$ for different $r_{s}: r_{s}=8.14, N_{p}=3, \eta=0.88(\bullet) ; r_{s}=5.76, N_{p}=6, \eta=0.77, M=27, N_{m}=1844$ (×) (compare with $(+)$ at smaller basis $\left.M=24, N_{m}=996, \eta=0.78\right) ; r_{s}=3.15, N_{p}=20$, $\eta=0.52(\mathrm{o})$; total statistics $N_{S}>2.5 \times 10^{4}$. Full lines show the Poisson distribution and the Wigner surmise. 


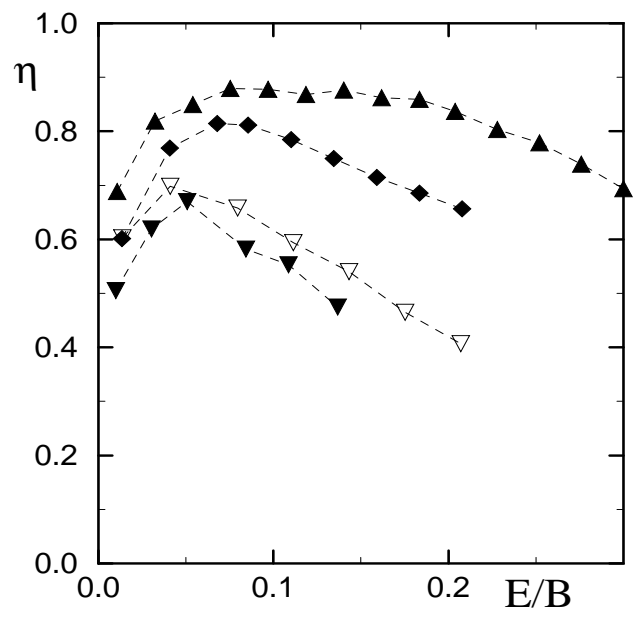

Fig. 4 Dependence of $\eta$ on $E / B$ for different $r_{s}$ at $L=25: r_{s}=8.14, N_{p}=3$ (full triangle up); $r_{s}=5.76, N_{p}=6$ (full diamond); $r_{s}=4.07, N_{p}=12$ (triangle down); $r_{s}=3.15$, $N_{p}=20$ (full triangle down).

only the interaction between two particles is able to mix many-body levels, the electron dynamics becomes nonergodic at large $r_{s}$ that leads to the Poisson distribution.

At the same time even at the optimal values of $r_{s} \approx 3$ the ergodicity and level mixing appear only at total energy $E>E_{c}$ while for $E<E_{c}$ including the ground state the ergodicity is absent in the range of parameters we studied. Formally, one can object against the statement that the value $\eta=1$ implies the localization of electrons in space giving as a counterexample the case of noninteracting delocalized electrons in the metallic phase where also $\eta=1$ for the multi-electron spectrum. However, in the system we study all one-particle states are exponentially localized and it is very difficult to imagine that the interaction gives delocalization of charge, makes the system metallic and does not introduce complete ergodicity and level mixing, which should give $\eta=0$ instead of $\eta=1$ found for $E<E_{c}$. Due to this reason we consider that the appearance of Poisson statistics near the ground state is the direct evidence of localization at $E<E_{c}$. At the same time the emergence of random matrix statistics at $E>E_{c}$ implies ergodicity and delocalization for many electron states. This delocalization apparently appears in a way similar to the two electron delocalization discussed in the previous section but in addition the interaction between larger number of particles decreases the delocalization border comparing to the TIP case discussed in [27, 28]. An interesting point is that the transition at $E=E_{c}$ takes place at zero temperature. Indeed, according to the data of Fig. 1 at the transition the energy per particle $\epsilon_{c}=E_{c} / N_{p} \approx 5 \times 10^{-3} B$ is much smaller than the Fermi energy $\epsilon_{F}=0.1 B$ showing that the data are close to the thermodynamic limit with $T=0$. 


\section{Conclusion}

Our results show that the Coulomb interaction between two electrons in excited states leads to their delocalization for $1<r_{s}<r_{L}^{4 / 3}$ while for $r_{s}>r_{L}^{4 / 3}$ they remain localized. The transition between these phases is similar to the Anderson transition in an effective dimension $d_{e f f}=3$. The numerical studies of the spectral statistics for many polarized electrons in the 2D Anderson model show that for $3<r_{s}<9$ and $5 \leq W / V \leq$ 15 the ground state is nonergodic (localized) and is characterized by the Poisson statistics for the total energy $E<E_{c}$. However, the transition to quantum ergodicity and the Wigner-Dyson statistics takes place at a fixed total energy $E_{c}$ independent of system size (for $r_{s} \approx 3.2,5 \leq W / V \leq 10$ and fixed filling $\nu \approx 1 / 32$ ). This implies a delocalization at zero temperature $T$. At the critical point $E_{c}$ the parameter $\eta_{c}$ increases with the disorder strength $W: \eta_{c}=0.19(W / V=5) ; 0.33(W / V=$ $7) ; 0.56(W / V=10)$ and the critical statistics approaches to the Poisson limit. In a certain sence the situation is similar to the Anderson transition in high dimensions $d>3$ discussed in [32] where also $\eta_{c}$ is growing with $d$. In analogy with this result and the case of two electrons in 2D we make a conjecture that in the Hamiltonian (1) the transition at $E_{c}$ is similar to a transition in some effective dimension $3 \leq d_{e f f}<2 N_{p}$. This $d_{\text {eff }}$ is growing with the disorder strength $W$.

The interaction induced ergodicity at $T=0$ is in favor of the metal-insulator transition observed experimentally, especially in the view of recently observed metallic behavior for polarized electrons [15]. However, our data are not sufficient to determine the behavior of resistivity on temperature in the ergodic phase at $E>E_{c}$. Therefore, it is not excluded that at strong disorder this ergodic phase will show a resistivity growth at low $T$. In this case one can suppose that the ergodicity induced by the Coulomb interaction is responsible for the phononless VRH conductivity as it was argued in [19, 18] and indicated by the experiments [16, 17]. More detailed investigations are required to understand the properties of the Coulomb ergodic phase at $E>E_{c}$.

We acknowledge the IDRIS at Orsay for the allocation of the CPU time on supercomputers.

\section{References}

[1] E. Abrahams, P. W. Anderson, D. C. Licciardello, and T. V. Ramakrishnan, Phys. Rev. Lett. 42 (1979) 673

[2] P. A. Lee, and T. V. Ramakhrishnan, Rev. Mod. Phys. 57 (1985) 287

[3] B. Kramer, and A. MacKinnon, Rep. Prog. Phys., 56 (1993) 1469

[4] S. V. Kravchenko, G. V. Kravchenko, J. E. Furneaux, V. M. Pudalov, and M. D'Iorio, Phys. Rev. B 50 (1994) 8039; S. V. Kravchenko, W. E. Mason, G. E. Bowker, J. E. Furneaux, V. M. Pudalov, and M. D'Iorio, Phys. Rev. B 51 (1995) 7038

[5] S. V. Kravchenko, D. Simonian, M. P. Sarachik, W. Mason, and J. E. Furneaux, Phys. Rev. Lett. 77 (1996) 4938

[6] D.Popović, A. B. Fowler, and S. Washburn, Phys. Rev. Lett. 79 (1997) 1543

[7] P. T. Coleridge, R. L. Williams, Y. Feng, and P. Zawadzki, Phys. Rev. B 56 (1997) R12764

[8] Y. Hanein, D. Shahar, J. Yoon, C. C. Li, D. C. Tsui, and H. Shtrikman, Phys. Rev. B 58 (1998) R7520

[9] M. Y. Simmons, A. R. Hamilton, M. Pepper, E. H. Linfield, P. D. Rose, and D. A. Ritchie, Phys. Rev. Lett. 80 (1998) 1292 
[10] V. M. Pudalov, G. Brunthaler, A.Prinz, and G. Bauer, JETP Lett. 68 (1998) 534

[11] V. Senz, U. Doetsch. U. Gepnser, T. Ihn, T. Heinzel, K. Ensslin, R. Hartmann, D. Gruetzmacher, cond-mat/9903367

[12] A. P. Mills, Jr., A. P. Ramirez, L. N. Pfeiffer, and K. W. West, cond-mat/9905176

[13] D. Simonian, S. V. Kravchenko, M. P. Sarachik, and V. M. Pudalov, Phys. Rev. Lett. 79 (1997) 2304

[14] J. Yoon, C. C. Li, D. Shahar, D. C. Tsui, and M. Shayegan, cond-mat/9907128v2

15] T. Okamoto, K. Hosoya, S. S. Kawaji, A. Yagi, A. Yutani, and Y. Shiraki, condmat/9906425

[16] W. Mason, S. V. Kravchenko, G. E. Bowker, and J. E. Furneaux, Phys. Rev. B 52 (1995) 7857

[17] S. I. Khondaker, I. S. Shlimak, J. T. Nicholls, M. Pepper, and D. A. Ritchie, Phys. Rev. B, 59 (1999) 4580

[18] D. G. Polyakov, and B. I. Shklovskii, Phys. Rev. B 48 (1993) 11167; I. L. Aleiner, D. G. Polyakov, and B. I. Shklovskii, Physics of semiconductors, World Scientific, Singapore, (1995) 787

[19] L. Fleishman, D. C. Licciardello, and P. W. Anderson, Phys. Rev. Lett. 40 (1978) 1340

[20] D. L. Shepelyansky, Phys. Rev. Lett. 73 (1994) 2607; p.201 in [25]

[21] Y. Imry, Europhys. Lett. 30 (1995) 405; p.211 in 25]

[22] D. Weinmann, A. Müller-Groeling, J.-L. Pichard, and K. Frahm, Phys. Rev. Lett. 75 (1995) 1598; p.221 in 25

[23] F. von Oppen, T. Wettig, and J. Müller, Phys. Rev. Lett. 76 (1996) 491; p. 235 in [25]

[24] Ph. Jacquod, and D. L. Shepelyansky, Phys. Rev. Lett. 78 (1997) 4986

[25] Correlated fermions and transport in mesoscopic systems, Eds. T.Martin, G.Montambaux and J.Trân Thanh Vân, Editions Frontieres, Gif-sur-Yvette, (1996)

[26] O. N. Dorokhov, Sov. Phys. JETP 71(2) (1990) 360

[27] D. L. Shepelyansky, cond-mat/9902246

[28] D. L. Shepelyansky, cond-mat/9905231

[29] P. H. Song and D. L. Shepelyansky, cond-mat/9904229

[30] B. I. Shklovskii, B. Shapiro, B. R. Sears, P. Lambrianides and H. B. Shore, Phys. Rev. B 47 (1993) 11487

[31] E. Cuevas, Phys. Rev. Lett. 83 (1999) 140; we note that for the short range interaction the majority of states is localized [20, 21] so that contrary to the claim of the paper $P(s) \rightarrow P_{P}(s)$ for large $L$ at any $W \neq 0$.

[32] I. Kh. Zharekeshev, and B. Kramer, Ann. Phys. (Leipzig) 7 (1998) 442; talk at this conference. 